\title{
Mathematical Properties of Variable Topological Indices
}

\author{
José M. Sigarreta
}

check for updates

Citation: Sigarreta, J.M. Mathematical Properties of Variable Topological Indices. Symmetry 2021, 13, 43. https://doi.org/10.3390/sym13010043

Received: 17 November 2020 Accepted: 24 December 2020 Published: 30 December 2020

Publisher's Note: MDPI stays neutral with regard to jurisdictional clai$\mathrm{ms}$ in published maps and institutional affiliations.

Copyright: $\odot 2020$ by the authors. Licensee MDPI, Basel, Switzerland. This article is an open access article distributed under the terms and conditions of the Creative Commons Attribution (CC BY) license (https:// creativecommons.org/licenses/by/ $4.0 /)$.
Faculty of Mathematics, Autonomous University of Guerrero, Carlos E. Adame 5, Col. La Garita, Acapulco 39087, Guerrero, Mexico; josemariasigarretaalmira@hotmail.com; Tel.: +52-744-159-2272

\begin{abstract}
A topic of current interest in the study of topological indices is to find relations between some index and one or several relevant parameters and/or other indices. In this paper we study two general topological indices $A_{\alpha}$ and $B_{\alpha}$, defined for each graph $H=(V(H), E(H))$ by $A_{\alpha}(H)=$ $\sum_{i j \in E(H)} f\left(d_{i}, d_{j}\right)^{\alpha}$ and $B_{\alpha}(H)=\sum_{i \in V(H)} h\left(d_{i}\right)^{\alpha}$, where $d_{i}$ denotes the degree of the vertex $i$ and $\alpha$ is any real number. Many important topological indices can be obtained from $A_{\alpha}$ and $B_{\alpha}$ by choosing appropriate symmetric functions and values of $\alpha$. This new framework provides new tools that allow to obtain in a unified way inequalities involving many different topological indices. In particular, we obtain new optimal bounds on the variable Zagreb indices, the variable sum-connectivity index, the variable geometric-arithmetic index and the variable inverse sum indeg index. Thus, our approach provides both new tools for the study of topological indices and new bounds for a large class of topological indices. We obtain several optimal bounds of $A_{\alpha}$ (respectively, $B_{\alpha}$ ) involving $A_{\beta}$ (respectively, $B_{\beta}$ ). Moreover, we provide several bounds of the variable geometric-arithmetic index in terms of the variable inverse sum indeg index, and two bounds of the variable inverse sum indeg index in terms of the variable second Zagreb and the variable sum-connectivity indices.
\end{abstract}

Keywords: variable Zagreb indices; variable sum-connectivity index; variable geometric-arithmetic index; variable inverse sum indeg index

\section{Introduction}

Chemical compounds (like hydrocarbons) can be represented by means of graphs. A topological descriptor is a numerical value (or a set of numerical values) that encapsulates some property of that graph. Moreover, if the descriptor correlates with a certain molecular characteristic, it is said to be a topological index, and it can be employed to study physicochemical properties of chemical substances.

In mathematical chemistry the analytical and structural properties of topological indices have been studied in depth over the last years (see, e.g., [1-3]). The theoretical and practical interest of topological indices lies in the fact that they have become an important tool for the study of multiple practical problems in Computer Science ([4]), Physic ([5]), Ecology ([6]), among others.

Countless applications of topological indices have been reported, most of them concerned with exploring medicinal and pharmacological issues. A turning point in the mathematical examination of topological indices happened in the second half of the 1990s, when a significant and ever growing research field on this matter started, resulting in numerous publications. In this context, especially the papers of Erdös [7,8] should be mentioned.

The concept of variable molecular descriptors was proposed as a new way of characterizing heteroatoms in molecules (see $[9,10])$, but also to assess structural differences (see [11]). In [12] the authors tested the correlation abilities of several topological indices used in practice for the case of standard heats of formation and normal boiling points of octane isomers.

In the sequel $H=(V(H), E(H))$ denotes a finite simple graph where, $V(H)$ is the set of vertices and $E(H)$ is the set of edges. $d_{i}$ denotes the degree of the vertex $i$. In [13-15], the following variable Zabreb indices are studied: 


$$
\begin{gathered}
M_{1}^{\alpha}(H)=\sum_{i \in V(H)} d_{i}^{\alpha}, \\
M_{2}^{\alpha}(H)=\sum_{i j \in E(H)}\left(d_{i} d_{j}\right)^{\alpha},
\end{gathered}
$$

with $\alpha \in \mathbb{R}$. $M_{1}^{\alpha}$ is known as the variable first Zabreb index and $M_{2}^{\alpha}$ is the variable second Zabreb index.

Note that if we take $\alpha=2$, we obtain the well-known first Zagreb index $M_{1}$; if $\alpha=-1$, we obtain the inverse degree index

$$
I D(H)=\sum_{i \in V(H)} \frac{1}{d_{i}}=\sum_{i j \in E(H)}\left(\frac{1}{d_{i}^{2}}+\frac{1}{d_{j}^{2}}\right),
$$

$M_{1}^{3}$ is the forgotten index, etc. Moreover, if $\alpha=-1 / 2$, we obtain $M_{2}^{-1 / 2}$ the famous Randić index and $M_{2}^{1}$ is the second Zagreb index. It is noteworthy that $M_{2}^{\alpha}$ with $\alpha=-1$ (the modified Zagreb index ([16])) performs significantly better than $M_{2}^{-1 / 2}$.

Besides, variable Zagreb indices have shown their theoretical importance and applicability in mathematical chemistry (see [17]). The idea behind the variable molecular descriptors is that the variables are determined during the regression so that the standard error of estimate for a particular studied property is as small as possible. For application details and mathematical theory see [14,15,18-22].

In [23] is studied the variable sum-connectivity index

$$
\chi_{\alpha}(H)=\sum_{i j \in E(H)}\left(d_{i}+d_{j}\right)^{\alpha},
$$

with $\alpha \in \mathbb{R}$.

Note that $\sum_{i j \in E(H)}\left(d_{i}+d_{j}\right)=\sum_{i \in V(H)} d_{i}^{2}$, thus $\chi_{1}$ is the first Zagreb index $M_{1}, 2 \chi_{-1}$ is the harmonic index $H$ (see [24-28]), etc. Some relations connecting these indices are reported in [26].

In $[29,30]$ the variable geometric-arithmetic index is defined by

$$
G A_{\alpha}(H)=\sum_{i j \in E(H)}\left(\frac{2 \sqrt{d_{i} d_{j}}}{d_{i}+d_{j}}\right)^{\alpha},
$$

with $\alpha \in \mathbb{R}$.

Clearly, $G A_{1}(H)$ and $G A_{-1}(H)$ are the geometric-arithmetic index $G A(H)$, and the arithmetic-geometric index $A G(H)$, respectively.

In $[30,31]$ is introduced the variable inverse sum indeg index as

$$
\operatorname{ISI}_{\alpha}(H)=\sum_{i j \in E(H)}\left(\frac{d_{i} d_{j}}{d_{i}+d_{j}}\right)^{\alpha} .
$$

with $\alpha \in \mathbb{R}$.

Note that $I S I_{1}(H)$ is the standard inverse sum indeg index $I S I(H)$.

\section{Motivation and Contributions}

The main reason for use topological indices is to obtain prediction of some property of molecules (see, e.g., [11,12]). Therefore, given some fixed parameters, a natural problem is to find the graphs that minimize (or maximize) the value of a topological index on the set of graphs satisfying the restrictions given by the parameters (see, e.g., $[7,8]$ ). 
Let $f: \mathbb{Z}^{+} \times \mathbb{Z}^{+} \rightarrow(0, \infty)$ be any symmetric function $(f(x, y)=f(y, x)), h: \mathbb{Z}^{+} \rightarrow$ $(0, \infty)$ any function and $\alpha \in \mathbb{R}$. Many important topological indices can be defined as

$$
A_{\alpha}(H)=\sum_{i j \in E(H)} f\left(d_{i}, d_{j}\right)^{\alpha},
$$

or

$$
B_{\alpha}(H)=\sum_{i \in V(H)} h\left(d_{i}\right)^{\alpha}
$$

One of the main problems in the study of topological indices is to find bounds for them, since this kind of results provide interrelations between different topological indices:

Since topological indices correlate with chemical properties of compounds, finding the compound with extreme behavior for a certain property is equivalent to optimizing a topological index $I_{1}$ with a strong correlation with that property on the appropriate set of graphs $\mathcal{H}$. If the behavior of the graphs with respect to the index $I_{2}$ is determined, and there exists a known relation between $I_{1}$ and $I_{2}$, then a large amount of the graphs in $\mathcal{H}$ can be removed a priori before starting the study. This leads to a significant simplification in the optimization problem.

The main goal of this paper is to obtain optimal bounds for the general topological indices $A_{\alpha}$ and $B_{\alpha}$. We have seen that many important topological indices can be obtained from $A_{\alpha}$ and $B_{\alpha}$ by choosing appropriate values of $\alpha$ and functions $f$ and $h$ :

- $\quad$ if $h(t)=t$, then $B_{2}$ is the first Zagreb index $M_{1}$;

- $\quad$ if $h(t)=t$, then $B_{-1}$ is the inverse degree index $I D$;

- $\quad$ if $h(t)=t$, then $B_{3}$ is the forgotten index $F$;

- $\quad$ if $h(t)=t$, then $B_{\alpha}$ is the variable first Zagreb index $M_{1}^{\alpha}$;

- $\quad$ if $f(x, y)=x y$, then $A_{1}$ is the second Zagreb index $M_{2}$;

- if $f(x, y)=x y$, then $A_{-1 / 2}$ is the usual Randić index $M_{2}^{-1 / 2}$;

- $\quad$ if $f(x, y)=x y$, then $A_{-1}$ is the modified Zagreb index $M Z$;

- $\quad$ if $f(x, y)=x y$, then $A_{\alpha}$ is the variable second Zagreb index $M_{2}^{\alpha}$;

- if $f(x, y)=x+y$, then $A_{-1 / 2}$ is the sum-connectivity index $\chi$;

- if $f(x, y)=x+y$, then $2 A_{-1}$ is the harmonic index $H$;

- if $f(x, y)=x+y$, then $A_{\alpha}$ is the variable sum-connectivity index $\chi_{\alpha}$;

- if $f(x, y)=2 \sqrt{x y} /(x+y)$, then $A_{1}$ is the geometric-arithmetic index $G A$;

- if $f(x, y)=2 \sqrt{x y} /(x+y)$, then $A_{-1}$ is the arithmetic-geometric index $A G$;

- if $f(x, y)=2 \sqrt{x y} /(x+y)$, then $A_{\alpha}$ is the variable geometric-arithmetic index $G A_{\alpha}$;

- $\quad$ if $f(x, y)=x y /(x+y)$, then $A_{1}$ is the variable inverse sum indeg index ISI;

- if $f(x, y)=x y /(x+y)$, then $A_{\alpha}$ is the variable inverse sum indeg index $I S I_{\alpha}$.

Thus, each theorem in this paper about $A_{\alpha}$ and $B_{\alpha}$ is a result for each one of these indices. Moreover, for each fixed function $f$ (respectively, $h$ ), we obtain a result for each real value of $\alpha$.

Our approach provides both new tools for the study of topological indices and new bounds for a large class of topological indices. In particular, we obtain several optimal bounds of $A_{\alpha}$ (respectively, $B_{\alpha}$ ) involving $A_{\beta}$ (respectively, $B_{\beta}$ ), see Theorems 1,2 and 3 (respectively, Propositions 1, 2 and 3). Moreover, we provide several optimal bounds of $G A_{\alpha}$ in terms of $I S I_{-\alpha}$ (see Theorems 4 and 5), and two optimal bounds of $I S I_{\alpha}$ involving $M_{2}^{2 \alpha}$ and $\chi_{-2 \alpha}$ in Theorem 6.

\section{Bounds for the General Topological Indices $A_{\alpha}$ and $B_{\alpha}$}

Throughout this work, $H=(V(H), E(H))$ denotes a finite simple graph. We denote by $\Delta, \delta, n, m$ the maximum degree, the minimum degree and the cardinality of the set of vertices (order) and edges (size) of $H$, respectively.

A graph is regular if all its vertices have the same degree. A graph is bipartite if a set of vertices can be partitioned into two disjointed sets so that all its edges have one end in 
each of those sets. A graph is said to be biregular if a bipartite graph such that the vertices of one bipartition have degree $\delta$ and the vertices of the other bipartition have degree $\Delta$.

In [32] it is proved that, if $p_{1}, \ldots, p_{m}$ are positive numbers which $\sum_{i=1}^{m} p_{i}=1$ and $F$ is a real continuous function that is convex, then:

$$
F\left(\sum_{i=1}^{m} p_{i}\left(x_{i}\right)\right) \leq\left(\sum_{i=1}^{m} p_{i} F\left(x_{i}\right)\right) .
$$

Note that if $p_{1}=\frac{1}{m}, \ldots, p_{m}=\frac{1}{m}$, we obtain the following particular case of Jensen's inequality. This inequality will be a main ingredient in the proof of Theorem 1 below.

Lemma 1. Let be $F: \mathbb{R}_{+} \rightarrow \mathbb{R}_{+}$a convex function and $x_{1}, \ldots, x_{m}>0$, then

$$
F\left(\frac{x_{1}+\cdots+x_{m}}{m}\right) \leq \frac{1}{m}\left(F\left(x_{1}\right)+\cdots+F\left(x_{m}\right)\right) .
$$

Theorem 1. Consider a symmetric function $f: \mathbb{Z}^{+} \times \mathbb{Z}^{+} \rightarrow(0, \infty)$. If $H$ is a graph of size $m$ and $\alpha, \beta \in \mathbb{R}$ with $\alpha \beta>0$, then

$$
A_{\alpha}(H) \geq \frac{m^{\alpha / \beta+1}}{A_{-\beta}(H)^{\alpha / \beta}},
$$

and the bound is tight if $H$ is a regular or biregular graph.

Proof. By the premise of the theorem we have to $\alpha, \beta \in \mathbb{R}$ with $\alpha \beta>0$. Since $-\alpha / \beta<0$, we can consider the convex function $F(x)=x^{-\alpha / \beta}$. Lemma 1 gives

$$
\begin{aligned}
\frac{m^{\alpha / \beta}}{\left(\sum_{i j \in E(H)} f\left(d_{i}, d_{j}\right)^{-\beta}\right)^{\alpha / \beta}} & \leq \frac{1}{m} \sum_{i j \in E(H)}\left(f\left(d_{i}, d_{j}\right)^{-\beta}\right)^{-\alpha / \beta} \\
& =\frac{1}{m} \sum_{i j \in E(H)} f\left(d_{i}, d_{j}\right)^{\alpha}, \\
\frac{m^{\alpha / \beta+1}}{A_{-\beta}(H)^{\alpha / \beta}} & \leq A_{\alpha}(H) .
\end{aligned}
$$

If $H$ is regular or biregular with maximum degree $\Delta$ and minimum degree $\delta$, then

$$
\frac{m^{\alpha / \beta+1}}{A_{-\beta}(H)^{\alpha / \beta}}=\frac{m^{\alpha / \beta+1}}{\left(f(\Delta, \delta)^{-\beta} m\right)^{\alpha / \beta}}=f(\Delta, \delta)^{\alpha} m=A_{\alpha}(H) .
$$

The previous theorem shows some mathematical relationships for the general variable index $A_{\alpha}$. Note that this result has an important theoretical scope since it allows to find bounds for all possible topological indices that can be written as a symmetric function of two real variables $(f(x, y))$.

The argument in the proof of Theorem 1 allows to prove the following result.

Proposition 1. Consider a function $h: \mathbb{Z}^{+} \rightarrow(0, \infty)$. If $H$ is a graph of order $n$ vertices and $\alpha, \beta \in \mathbb{R}$ with $\alpha \beta>0$, then

$$
B_{\alpha}(H) \geq \frac{n^{\alpha / \beta+1}}{B_{-\beta}(H)^{\alpha / \beta}}
$$

and the bound is tight if $\mathrm{H}$ is a regular graph. 
The previous proposition shows some mathematical relations for the general variable index $B_{\alpha}$. This result has an important theoretical scope since it allows to find bounds for all possible topological indices that can be written as a function of a real variable $(h(t))$.

Corollary 1. Let $H$ be a graph of order $n$ and size $m$, and let $\alpha, \beta \in \mathbb{R}$ with $\alpha \beta>0$. Then

$$
\begin{array}{cc}
M_{2}^{\alpha}(H) \geq \frac{m^{\alpha / \beta+1}}{M_{2}^{-\beta}(H)^{\alpha / \beta}}, & \chi_{\alpha}(H) \geq \frac{m^{\alpha / \beta+1}}{\chi_{-\beta}(H)^{\alpha / \beta}}, \\
G A_{\alpha}(H) \geq \frac{m^{\alpha / \beta+1}}{G A_{-\beta}(H)^{\alpha / \beta}}, & I S I_{\alpha}(H) \geq \frac{m^{\alpha / \beta+1}}{I S I_{-\beta}(H)^{\alpha / \beta}},
\end{array}
$$

the equality in each bound is attained if $H$ is a regular or biregular graph. Moreover,

$$
M_{1}^{\alpha}(H) \geq \frac{n^{\alpha / \beta+1}}{M_{1}^{-\beta}(H)^{\alpha / \beta}}
$$

the equality in the bound is attained if $H$ is a regular graph.

Proof. Let $f_{1}, f_{2}, f_{3}, f_{4}: \mathbb{Z}^{+} \times \mathbb{Z}^{+} \rightarrow(0, \infty)$ defined as

$$
f_{1}(x, y)=x y, \quad f_{2}(x, y)=x+y, \quad f_{3}(x, y)=\frac{2 \sqrt{x y}}{x+y}, \quad f_{4}(x, y)=\frac{x y}{x+y},
$$

it is clear that these are symmetric functions, then applying Theorem 1 with the functions $f_{1}, f_{2}, f_{3}, f_{4}$ respectively we have

$$
\begin{array}{cc}
M_{2}^{\alpha}(H) \geq \frac{m^{\alpha / \beta+1}}{M_{2}^{-\beta}(H)^{\alpha / \beta}}, & \chi_{\alpha}(H) \geq \frac{m^{\alpha / \beta+1}}{\chi_{-\beta}(H)^{\alpha / \beta}}, \\
G A_{\alpha}(H) \geq \frac{m^{\alpha / \beta+1}}{G A_{-\beta}(H)^{\alpha / \beta}}, & I S I_{\alpha}(H) \geq \frac{m^{\alpha / \beta+1}}{I S I_{-\beta}(H)^{\alpha / \beta}},
\end{array}
$$

and the equality in each bound is attained if $H$ is a regular or biregular graph, let $h: \mathbb{Z}^{+} \rightarrow$ $(0, \infty)$ defined as $h(x)=x$ then by Proposition 1 we have

$$
M_{1}^{\alpha}(H) \geq \frac{n^{\alpha / \beta+1}}{M_{1}^{-\beta}(H)^{\alpha / \beta}}
$$

and the equality in the bound is attained if $H$ is a regular graph.

The previous corollary establishes new relations between some variable topological indices which are common in many theoretical and practical applications.

Theorem 2. Consider a symmetric function $f: \mathbb{Z}^{+} \times \mathbb{Z}^{+} \rightarrow(0, \infty)$. If $H$ is a graph of size $m$ and $\alpha, \beta \in \mathbb{R}$ with $\alpha \beta>0$ and $|\beta| \leq|\alpha|$, then

$$
A_{\alpha}(H) \geq m^{1-\alpha / \beta} A_{\beta}(H)^{\alpha / \beta}
$$

and the bound is tight if $H$ is a regular or biregular graph. 
Proof. By the premise of the theorem we have to $\alpha, \beta \in \mathbb{R}$ with $\alpha \beta>0$. Since $\alpha / \beta \geq 1$, we can consider the convex function $F(x)=x^{\alpha / \beta}$. Lemma 1 gives

$$
\begin{aligned}
\left(\frac{1}{m} \sum_{i j \in E(H)} f\left(d_{i}, d_{j}\right)^{\beta}\right)^{\alpha / \beta} & \leq \frac{1}{m} \sum_{i j \in E(H)}\left(f\left(d_{i}, d_{j}\right)^{\beta}\right)^{\alpha / \beta} \\
& =\frac{1}{m} \sum_{i j \in E(H)} f\left(d_{i}, d_{j}\right)^{\alpha}, \\
m^{1-\alpha / \beta} A_{\beta}(H)^{\alpha / \beta} & \leq A_{\alpha}(H) .
\end{aligned}
$$

If $H$ is regular or biregular with maximum degree $\Delta$ and minimum degree $\delta$, then

$$
m^{1-\alpha / \beta} A_{\beta}(H)^{\alpha / \beta}=m^{1-\alpha / \beta}\left(f(\Delta, \delta)^{\beta} m\right)^{\alpha / \beta}=f(\Delta, \delta)^{\alpha} m=A_{\alpha}(H) .
$$

The previous theorem also shows some mathematical relationships for the general variable index $A_{\alpha}$. Note that this result has an important theoretical scope since it allows to find bounds for all possible topological indices that can be written as a symmetric function of two real variables.

Proposition 2. Consider a function $h: \mathbb{Z}^{+} \rightarrow(0, \infty)$. If $H$ is a graph of order $n$ and $\alpha, \beta \in \mathbb{R}$ with $\alpha \beta>0$ and $|\beta| \leq|\alpha|$, then

$$
B_{\alpha}(H) \geq n^{1-\alpha / \beta} B_{\beta}(H)^{\alpha / \beta},
$$

and the bound is tight if $\mathrm{H}$ is a regular graph.

Proof. Since $\alpha / \beta \geq 1$, we can consider the convex function $F(x)=x^{\alpha / \beta}$. Lemma 1 gives

$$
\begin{aligned}
\left(\frac{1}{m} \sum_{i \in V(H)} h\left(d_{i}\right)^{\beta}\right)^{\alpha / \beta} & \leq \frac{1}{n} \sum_{i \in V(H)}\left(h\left(d_{i}\right)^{\beta}\right)^{\alpha / \beta} \\
& =\frac{1}{n} \sum_{i \in V(H)} h\left(d_{i}\right)^{\alpha}, \\
n^{1-\alpha / \beta} B_{\beta}(H)^{\alpha / \beta} & \leq B_{\alpha}(H) .
\end{aligned}
$$

If $H$ is regular, then $d_{i}=\delta$ for all $i \in V(H)$ and

$$
n^{1-\alpha / \beta} B_{\beta}(H)^{\alpha / \beta}=n^{1-\alpha / \beta}\left(h(\delta)^{\beta} n\right)^{\alpha / \beta}=h(\delta)^{\alpha} n=B_{\alpha}(H) .
$$

The previous proposition shows some mathematical relations for the general variable index $B_{\alpha}$. This result has an important theoretical scope since it allows to find bounds for all possible topological indices that can be written as a function of a real variable.

The proof of the following corollary is analogous to the proof of Corollary 1 using the results obtained in Theorem 2 and Proposition 2.

Corollary 2. If $H$ is a graph of order $n$, size $m$ and $\alpha, \beta \in \mathbb{R}$ with $\alpha \beta>0$ and $|\beta| \leq|\alpha|$, then

$$
\begin{array}{cl}
M_{2}^{\alpha}(H) \geq m^{1-\alpha / \beta} M_{2}^{\beta}(H)^{\alpha / \beta}, & \chi_{\alpha}(H) \geq m^{1-\alpha / \beta} \chi_{\beta}(H)^{\alpha / \beta}, \\
G A_{\alpha}(H) \geq m^{1-\alpha / \beta} G A_{\beta}(H)^{\alpha / \beta}, & \operatorname{ISI}_{\alpha}(H) \geq m^{1-\alpha / \beta} \operatorname{ISI}_{\beta}(H)^{\alpha / \beta},
\end{array}
$$


the bound is tight if $H$ is a regular or biregular graph,

$$
M_{1}^{\alpha}(H) \geq n^{1-\alpha / \beta} M_{1}^{\beta}(H)^{\alpha / \beta},
$$

the bound is tight if $H$ is a regular graph.

Note that the previous corollary provides optimal bounds for variable topological indices based on two important invariants of a graph (maximum degree and minimum degree).

The following Lemma appears in [22]. This inequality will be useful in the proofs of Theorem 3 and Proposition 3 below.

Lemma 2. Consider real numbers $0<\beta \leq 1 \leq \alpha, M>0$ and $0 \leq x_{1}, \ldots, x_{n} \leq M$. Then

$$
\left(\sum_{j=1}^{n} x_{j}^{\alpha}\right)^{1 / \alpha} \leq M^{1-\beta} \sum_{j=1}^{n} x_{j}^{\beta}
$$

Theorem 3. Consider a symmetric function $f: \mathbb{Z}^{+} \times \mathbb{Z}^{+} \rightarrow(0, \infty)$ and $\alpha, \beta \in \mathbb{R}$. If $H$ is a graph with maximum degree $\Delta$ and minimum degree $\delta$, and

$$
\begin{aligned}
& F_{\Delta, \delta}=\max \left\{f(x, y): x, y \in \mathbb{Z}^{+}, \delta \leq x, y \leq \Delta\right\}, \\
& f_{\Delta, \delta}=\min \left\{f(x, y): x, y \in \mathbb{Z}^{+}, \delta \leq x, y \leq \Delta\right\},
\end{aligned}
$$

then

$$
\begin{array}{cl}
A_{\alpha}(H) \leq F_{\Delta, \delta}^{\alpha(1-\beta)} A_{\beta}(H)^{\alpha}, & \text { if } 0<\beta \leq 1 \leq \alpha, \\
A_{\alpha}(H) \leq f_{\Delta, \delta}^{\alpha(1+\beta)} A_{\beta}(H)^{-\alpha}, & \text { if } \alpha \leq-1 \leq \beta<0 .
\end{array}
$$

Proof. Let us consider first the case $0<\beta \leq 1 \leq \alpha$. Since $f\left(d_{i}, d_{j}\right) \leq F_{\Delta, \delta}$ for every $i j \in E(H)$, Lemma 2 gives

$$
\left(\sum_{i j \in E(H)}^{n} f\left(d_{i}, d_{j}\right)^{\alpha}\right)^{1 / \alpha} \leq F_{\Delta, \delta}^{1-\beta} \sum_{i j \in E(H)}^{n} f\left(d_{i}, d_{j}\right)^{\beta}
$$

Consider now the case $\alpha \leq-1 \leq \beta<0$ and define $a=-\alpha$ and $b=-\beta$. Thus, $0<b \leq 1 \leq a$. If we consider the function $1 / f$, then $1 / f\left(d_{i}, d_{j}\right) \leq 1 / f_{\Delta, \delta}$ for every $i j \in E(H)$, and the previous argument gives

$$
\begin{gathered}
\left(\sum_{i j \in E(H)}^{n} \frac{1}{f\left(d_{i}, d_{j}\right)^{a}}\right)^{1 / a} \leq \frac{1}{f_{\Delta, \delta}^{1-b}} \sum_{i j \in E(H)}^{n} \frac{1}{f\left(d_{i}, d_{j}\right)^{b}}, \\
\left(\sum_{i j \in E(H)}^{n} f\left(d_{i}, d_{j}\right)^{\alpha}\right)^{-1 / \alpha} \leq f_{\Delta, \delta}^{-1-\beta} \sum_{i j \in E(H)}^{n} f\left(d_{i}, d_{j}\right)^{\beta} .
\end{gathered}
$$

The previous theorem shows some mathematical relationships for the general variable index $A_{\alpha}$. Note that this result has an important theoretical scope since it allows to find bounds for all possible topological indices that can be written as a symmetric function of two real variables.

Proposition 3. Consider a function $h: \mathbb{Z}^{+} \rightarrow(0, \infty)$ and $\alpha, \beta \in \mathbb{R}$. If $H$ is a graph with maximum degree $\Delta$ and minimum degree $\delta$, and

$$
\begin{aligned}
K_{\Delta, \delta} & =\max \left\{h(x): x \in \mathbb{Z}^{+}, \delta \leq x \leq \Delta\right\}, \\
k_{\Delta, \delta} & =\min \left\{h(x): x \in \mathbb{Z}^{+}, \delta \leq x \leq \Delta\right\},
\end{aligned}
$$


then

$$
\begin{array}{cl}
B_{\alpha}(H) \leq K_{\Delta, \delta}^{\alpha(1-\beta)} B_{\beta}(H)^{\alpha}, & \text { if } 0<\beta \leq 1 \leq \alpha, \\
B_{\alpha}(H) \leq k_{\Delta, \delta}^{\alpha(1+\beta)} B_{\beta}(H)^{-\alpha}, & \text { if } \alpha \leq-1 \leq \beta<0 .
\end{array}
$$

Proof. Let us consider first the case $0<\beta \leq 1 \leq \alpha$. Since $h\left(d_{i}\right) \leq K_{\Delta, \delta}$ for every $i \in V(H)$, Lemma 2 gives

$$
\left(\sum_{i \in V(H)}^{n} h\left(d_{i}\right)^{\alpha}\right)^{1 / \alpha} \leq K_{\Delta, \delta}^{1-\beta} \sum_{i \in V(H)}^{n} h\left(d_{i}\right)^{\beta} .
$$

Consider now the case $\alpha \leq-1 \leq \beta<0$ and define $a=-\alpha$ and $b=-\beta$. Thus, $0<b \leq 1 \leq a$. If we consider the function $1 / h$, then $1 / h\left(d_{i}\right) \leq 1 / k_{\Delta, \delta}$ for every $i \in V(H)$, and the previous argument gives

$$
\begin{gathered}
\left(\sum_{i \in V(H)}^{n} \frac{1}{h\left(d_{i}\right)^{a}}\right)^{1 / a} \leq \frac{1}{k_{\Delta, \delta}^{1-b}} \sum_{i \in V(H)}^{n} \frac{1}{f\left(d_{i}\right)^{b}}, \\
\left(\sum_{i \in V(H)}^{n} h\left(d_{i}\right)^{\alpha}\right)^{-1 / \alpha} \leq k_{\Delta, \delta}^{-1-\beta} \sum_{i \in V(H)}^{n} f\left(d_{i}\right)^{\beta} .
\end{gathered}
$$

The previous proposition also shows some mathematical relations for the general variable index $B_{\alpha}$. This result has an important theoretical scope since it allows to find bounds for all possible topological indices that can be written as a function of a real variable.

The proof of the following corollary is quite analogous to the proof of Corollary 1 using the results obtained in Theorem 3 and Proposition 3.

Corollary 3. Consider a symmetric function $f: \mathbb{Z}^{+} \times \mathbb{Z}^{+} \rightarrow(0, \infty)$ and $\alpha, \beta \in \mathbb{R}$. If $H$ is a graph with minimum degree $\delta$ and maximum degree $\Delta$, then

$$
\begin{array}{cl}
M_{2}^{\alpha}(H) \leq \Delta^{2 \alpha(1-\beta)} M_{2}^{\beta}(H)^{\alpha}, & \text { if } 0<\beta \leq 1 \leq \alpha, \\
M_{2}^{\alpha}(H) \leq \delta^{2 \alpha(1+\beta)} M_{2}^{\beta}(H)^{-\alpha}, & \text { if } \alpha \leq-1 \leq \beta<0 . \\
\chi_{\alpha}(H) \leq(2 \Delta)^{\alpha(1-\beta)} \chi_{\beta}(H)^{\alpha}, & \text { if } 0<\beta \leq 1 \leq \alpha, \\
\chi_{\alpha}(H) \leq(2 \delta)^{\alpha(1+\beta)} \chi_{\beta}(H)^{-\alpha}, & \text { if } \alpha \leq-1 \leq \beta<0 . \\
G A_{\alpha}(H) \leq G A_{\beta}(H)^{\alpha}, & \text { if } 0<\beta \leq 1 \leq \alpha, \\
G A_{\alpha}(H) \leq\left(\frac{2 \sqrt{\Delta \delta}}{\Delta+\delta}\right)^{\alpha(1+\beta)} G_{\beta}(H)^{-\alpha}, & \text { if } \alpha \leq-1 \leq \beta<0 . \\
\text { ISI }_{\alpha}(H) \leq\left(\frac{\Delta}{2}\right)^{\alpha(1-\beta)} \operatorname{ISI}_{\beta}(H)^{\alpha}, & \text { if } 0<\beta \leq 1 \leq \alpha, \\
\text { ISI }_{\alpha}(H) \leq\left(\frac{\delta}{2}\right)^{\alpha(1+\beta)} \operatorname{ISI}_{\beta}(H)^{-\alpha}, & \text { if } \alpha \leq-1 \leq \beta<0 .
\end{array}
$$

The previous results establish new bounds on the variable topological indices. Furthermore, in this same direction, the well known classical indices (harmonic index, Randić index, Zagred index, among others) appear as particular cases $A_{\alpha}$ and $B_{\alpha}$.

\section{Relations between $G A_{\alpha}$ and $I S I_{\alpha}$}

Theorem 4. If $H$ is a graph with minimum degree $\delta$, maximum degree $\Delta$ and $\alpha>0$, then

$$
\left(\frac{2}{\Delta}\right)^{\alpha} \operatorname{ISI}_{\alpha}(H) \leq G A_{-\alpha}(H) \leq\left(\max \left\{\frac{2}{\delta}, \frac{(\Delta+\delta)^{2}}{2 \sqrt{\Delta^{3} \delta^{3}}}\right\}\right)^{\alpha} \operatorname{ISI}_{\alpha}(H) .
$$


The lower bound is tight if and only if $H$ is regular. If $\frac{2}{\delta}>\frac{(\Delta+\delta)^{2}}{2 \sqrt{\Delta^{3} \delta^{3}}}$, the upper bound is tight if and only if $H$ is regular. If $\frac{2}{\delta}<\frac{(\Delta+\delta)^{2}}{2 \sqrt{\Delta^{3} \delta^{3}}}$, the upper bound is tight if and only if $H$ is biregular. If $\frac{2}{\delta}=\frac{(\Delta+\delta)^{2}}{2 \sqrt{\Delta^{3} \delta^{3}}}$, the upper bound is tight if and only if $H$ is regular or biregular.

Proof. We are going to study the function

$$
P(x, y)=\frac{x+y}{2 \sqrt{x y}} \frac{x+y}{x y}=\frac{1}{2}(x+y)^{2} x^{-3 / 2} y^{-3 / 2},
$$

on the set $\{\delta \leq x \leq y \leq \Delta\}$. Since

$$
\begin{aligned}
\frac{\partial P}{\partial x}(x, y) & =\frac{1}{2} y^{-3 / 2}\left[2(x+y) x^{-3 / 2}\left(-\frac{3}{2}\right)(x+y)^{2} x^{-5 / 2}\right] \\
& =\frac{1}{2} y^{-3 / 2} x^{-5 / 2}(x+y)\left[2 x\left(-\frac{3}{2}\right)(x+y)\right] \\
& =\frac{1}{4} y^{-3 / 2} x^{-5 / 2}(x+y)(x-3 y) \leq 0,
\end{aligned}
$$

so $P(x, y)$ is a strictly decreasing function on $x \in[\delta, y]$ for each fixed $y \in[\delta, \Delta]$, and so $P(y, y) \leq P(x, y) \leq P(\delta, y)$.

Since $P(y, y)=2 / y$ is a strictly decreasing function on $y \in[\delta, \Delta]$, we have $\frac{2}{\Delta}=$ $P(\Delta, \Delta) \leq P(x, y)$ and the equality is attained if and only if $x=y=\Delta$. Thus,

$$
\begin{aligned}
\left(\frac{2}{\Delta}\right)^{\alpha}\left(\frac{d_{i} d_{j}}{d_{i}+d_{j}}\right)^{\alpha} & \leq\left(\frac{d_{i}+d_{j}}{2 \sqrt{d_{i} d_{j}}}\right)^{\alpha} \quad \text { for any } i j \in E(H), \\
\left(\frac{2}{\Delta}\right)^{\alpha} I S I_{\alpha}(H) & \leq G A_{-\alpha}(H),
\end{aligned}
$$

and this last bond is tight if and only if $d_{i}=d_{j}=\Delta$ for each $i j \in E(H)$, i.e., $H$ is a regular graph.

Define

$$
p(y)=P(\delta, y)=\frac{1}{2}(y+\delta)^{2} y^{-3 / 2} \delta^{-3 / 2} .
$$

We have

$$
\begin{aligned}
p^{\prime}(y) & =\frac{1}{2} \delta^{-3 / 2}\left[2(y+\delta) y^{-3 / 2}+\left(-\frac{3}{2}\right)(y+\delta)^{2} y^{-5 / 2}\right] \\
& =\frac{1}{2} \delta^{-3 / 2} y^{-5 / 2}(y+\delta)\left[2 y+\left(-\frac{3}{2}\right)(y+\delta)\right] \\
& =\frac{1}{4} \delta^{-3 / 2} y^{-5 / 2}(y+\delta)(y-3 \delta) .
\end{aligned}
$$

Since $p^{\prime}(y)$ has at most a zero on $[\delta, \Delta]$ and $p^{\prime}(\delta)<0$, we conclude

$$
\max _{y \in[\delta, \Delta]} p(y)=\max \{p(\delta), p(\Delta)\}
$$

Thus,

$$
P(x, y) \leq P(\delta, y)=p(y) \leq \max \left\{\frac{2}{\delta}, \frac{(\Delta+\delta)^{2}}{2 \sqrt{\Delta^{3} \delta^{3}}}\right\} .
$$

If $\frac{2}{\delta}>\frac{(\Delta+\delta)^{2}}{2 \sqrt{\Delta^{3} \delta^{3}}}$, then the equality in the bound holds if and only if $x=y=\delta$, if $\frac{2}{\delta}<\frac{(\Delta+\delta)^{2}}{2 \sqrt{\Delta^{3} \delta^{3}}}$, then the equality in the bound holds if and only if $x=\delta$ and $y=\Delta$, if 
$\frac{2}{\delta}=\frac{(\Delta+\delta)^{2}}{2 \sqrt{\Delta^{3} \delta^{3}}}$, then the equality in the bound holds if and only if $x=y=\delta$ or $x=\delta$ and $y=\Delta$. Therefore,

$$
\begin{aligned}
\left(\frac{d_{i}+d_{j}}{2 \sqrt{d_{i} d_{j}}}\right)^{\alpha} & \leq\left(\max \left\{\frac{2}{\delta}, \frac{(\Delta+\delta)^{2}}{2 \sqrt{\Delta^{3} \delta^{3}}}\right\}\right)^{\alpha}\left(\frac{d_{i} d_{j}}{d_{i}+d_{j}}\right)^{\alpha} \text { for any } i j \in E(H), \\
G A_{-\alpha}(H) & \leq\left(\max \left\{\frac{2}{\delta}, \frac{(\Delta+\delta)^{2}}{2 \sqrt{\Delta^{3} \delta^{3}}}\right\}\right)^{\alpha} \operatorname{ISI}_{\alpha}(H) .
\end{aligned}
$$

If $\frac{2}{\delta}>\frac{(\Delta+\delta)^{2}}{2 \sqrt{\Delta^{3} \delta^{3}}}$, then the bound is tight if and only if $d_{i}=d_{j}=\delta$ for any $i j \in E(H)$, i.e., $H$ is regular. If $\frac{2}{\delta}<\frac{(\Delta+\delta)^{2}}{2 \sqrt{\Delta^{3} \delta^{3}}}$, then the bound is tight if and only if $\left\{d_{i}, d_{j}\right\}=\{\delta, \Delta\}$ for any $i j \in E(H)$, i.e., $H$ is biregular. If $\frac{2}{\delta}=\frac{(\Delta+\delta)^{2}}{2 \sqrt{\Delta^{3} \delta^{3}}}$, then the bound is tight if and only if $d_{i}=d_{j}=\delta$ for any $i j \in E(H)$, or $\left\{d_{i}, d_{j}\right\}=\{\delta, \Delta\}$ for every $i j \in E(H)$, i.e., $H$ is regular or biregular.

The previous theorem shows some relations between the variable geometric-arithmetic index $G A_{\alpha}$ and the variable inverse sum indeg index $I S I_{\alpha}$, which are two of the most important and well-studied variable topological indices.

Theorem 5. If $H$ is a graph with minimum degree $\delta$, maximum degree $\Delta$ and $\alpha>0$, then

$$
\left(\min \left\{\frac{\delta}{2}, \frac{2 \sqrt{\Delta^{3} \delta^{3}}}{(\Delta+\delta)^{2}}\right\}\right)^{\alpha} I S I_{-\alpha}(H) \leq G A_{\alpha}(H) \leq\left(\frac{\Delta}{2}\right)^{\alpha} I S I_{-\alpha}(H) .
$$

The upper bound is tight if and only if $H$ is regular. If $\frac{\delta}{2}<\frac{2 \sqrt{\Delta^{3} \delta^{3}}}{(\Delta+\delta)^{2}}$, the lower bound is tight if and only if $H$ is regular. If $\frac{\delta}{2}>\frac{2 \sqrt{\Delta^{3} \delta^{3}}}{(\Delta+\delta)^{2}}$, the lower bound is tight if and only if $H$ is biregular. If $\frac{\delta}{2}=\frac{2 \sqrt{\Delta^{3} \delta^{3}}}{(\Delta+\delta)^{2}}$, the lower bound is tight if and only if $H$ is regular or biregular.

Proof. The argument in the proof of Theorem 4 gives that

$$
\frac{2}{\Delta} \frac{d_{i} d_{j}}{d_{i}+d_{j}} \leq \frac{d_{i}+d_{j}}{2 \sqrt{d_{i} d_{j}}} \leq \max \left\{\frac{2}{\delta}, \frac{(\Delta+\delta)^{2}}{2 \sqrt{\Delta^{3} \delta^{3}}}\right\} \frac{d_{i} d_{j}}{d_{i}+d_{j}}
$$

for every $i j \in E(H)$. Hence,

$$
\left(\frac{2}{\Delta}\right)^{-\alpha}\left(\frac{d_{i} d_{j}}{d_{i}+d_{j}}\right)^{-\alpha} \geq\left(\frac{2 \sqrt{d_{i} d_{j}}}{d_{i}+d_{j}}\right)^{\alpha} \geq\left(\max \left\{\frac{2}{\delta}, \frac{(\Delta+\delta)^{2}}{2 \sqrt{\Delta^{3} \delta^{3}}}\right\}\right)^{-\alpha}\left(\frac{d_{i} d_{j}}{d_{i}+d_{j}}\right)^{-\alpha}
$$

and so,

$$
\left(\min \left\{\frac{\delta}{2}, \frac{2 \sqrt{\Delta^{3} \delta^{3}}}{(\Delta+\delta)^{2}}\right\}\right)^{\alpha}\left(\frac{d_{i} d_{j}}{d_{i}+d_{j}}\right)^{-\alpha} \leq\left(\frac{2 \sqrt{d_{i} d_{j}}}{d_{i}+d_{j}}\right)^{\alpha} \leq\left(\frac{\Delta}{2}\right)^{\alpha}\left(\frac{d_{i} d_{j}}{d_{i}+d_{j}}\right)^{-\alpha}
$$

for any $i j \in E(H)$, and

$$
\left(\min \left\{\frac{\delta}{2}, \frac{2 \sqrt{\Delta^{3} \delta^{3}}}{(\Delta+\delta)^{2}}\right\}\right)^{\alpha} I S I_{-\alpha}(H) \leq G A_{\alpha}(H) \leq\left(\frac{\Delta}{2}\right)^{\alpha} I S I_{-\alpha}(H) .
$$


The previous theorem is useful, since it provides functional properties of the variable topological indices $G A_{\alpha}$ and $I S I_{-\alpha}$, and two important invariants of a graph (the maximum degree $\Delta$ and the minimum degree $\delta$ ).

The following Lemma is well-known (see, e.g., ([33], Lemma 5) for a proof of the equality statement). It will be useful in the proof of Theorem 6 below.

Lemma 3. If $a_{i}, b_{i} \geq 0$ and $\omega b_{i} \leq a_{i} \leq \Omega b_{i}$ for $1 \leq i \leq n$, then

$$
\left(\sum_{i=1}^{n} a_{i}^{2}\right)\left(\sum_{i=1}^{n} b_{i}^{2}\right) \leq \frac{(\Omega+\omega)^{2}}{4 \Omega \omega}\left(\sum_{i=1}^{n} a_{i} b_{i}\right)^{2} .
$$

If $a_{i}>0$ for some $1 \leq i \leq n$, then the equality holds if and only if $\omega=\Omega$ and $a_{i}=\omega b_{i}$ for every $1 \leq i \leq n$.

Theorem 6. If $H$ is a graph with minimum degree $\delta$, maximum degree $\Delta$ and $\alpha>0$, then

$$
\frac{2 \Delta^{3 \alpha / 2} \delta^{3 \alpha / 2}}{\Delta^{3 \alpha}+\delta^{3 \alpha}} \sqrt{M_{2}^{2 \alpha}(H) \chi_{-2 \alpha}(H)} \leq \operatorname{ISI}_{\alpha}(H) \leq \sqrt{M_{2}^{2 \alpha}(H) \chi_{-2 \alpha}(H)} .
$$

Moreover, the lower bound is tight if and only if $\mathrm{H}$ is regular, while the upper bound is tight if $H$ is a regular or biregular graph.

Proof. Cauchy-Schwarz inequality gives

$$
\begin{aligned}
\operatorname{ISI}_{\alpha}(H)^{2} & =\left(\sum_{i j \in E(H)}\left(d_{i} d_{j}\right)^{\alpha} \frac{1}{\left(d_{i}+d_{j}\right)^{\alpha}}\right)^{2} \\
& \leq \sum_{i j \in E(H)}\left(d_{i} d_{j}\right)^{2 \alpha} \sum_{i j \in E(H)} \frac{1}{\left(d_{i}+d_{j}\right)^{2 \alpha}}=M_{2}^{2 \alpha}(H) \chi_{-2 \alpha}(H) .
\end{aligned}
$$

If $H$ is a regular or biregular graph, then

$$
\sqrt{M_{2}^{2 \alpha}(H) \chi_{-2 \alpha}(H)}=\sqrt{(\Delta \delta)^{2 \alpha} m \frac{m}{(\Delta+\delta)^{2 \alpha}}}=\frac{(\Delta \delta)^{\alpha}}{(\Delta+\delta)^{\alpha}} m=\operatorname{ISI}_{\alpha}(H) .
$$

Let us prove the lower bound.

If $\alpha \geq 0$, then

$$
2^{\alpha} \delta^{3 \alpha} \leq\left(d_{i} d_{j}\right)^{\alpha}\left(d_{i}+d_{j}\right)^{\alpha}=\frac{\left(d_{i} d_{j}\right)^{\alpha}}{\frac{1}{\left(d_{i}+d_{j}\right)^{\alpha}}} \leq 2^{\alpha} \Delta^{3 \alpha} .
$$

If $\alpha<0$, then

$$
2^{\alpha} \Delta^{3 \alpha} \leq\left(d_{i} d_{j}\right)^{\alpha}\left(d_{i}+d_{j}\right)^{\alpha}=\frac{\left(d_{i} d_{j}\right)^{\alpha}}{\frac{1}{\left(d_{i}+d_{j}\right)^{\alpha}}} \leq 2^{\alpha} \delta^{3 \alpha} .
$$

Since

$$
\frac{\Omega+\omega}{2 \sqrt{\Omega \omega}}=\frac{1}{2} \sqrt{\frac{\Omega}{\omega}}+\frac{1}{2} \sqrt{\frac{\omega}{\Omega}}=\frac{1}{2} \sqrt{\frac{2^{\alpha} \Delta^{3 \alpha}}{2^{\alpha} \delta^{3 \alpha}}}+\frac{1}{2} \sqrt{\frac{2^{\alpha} \delta^{3 \alpha}}{2^{\alpha} \Delta^{3 \alpha}}}=\frac{\Delta^{3 \alpha}+\delta^{3 \alpha}}{2 \Delta^{3 \alpha / 2} \delta^{3 \alpha / 2}}
$$

for every $\alpha \in \mathbb{R}$, Lemma 3 gives that 


$$
\begin{aligned}
\operatorname{ISI}_{\alpha}(H)^{2} & =\left(\sum_{i j \in E(H)}\left(d_{i} d_{j}\right)^{\alpha} \frac{1}{\left(d_{i}+d_{j}\right)^{\alpha}}\right)^{2} \\
& \geq\left(\frac{2 \Delta^{3 \alpha / 2} \delta^{3 \alpha / 2}}{\Delta^{3 \alpha}+\delta^{3 \alpha}}\right)^{2} \sum_{i j \in E(H)}\left(d_{i} d_{j}\right)^{2 \alpha} \sum_{i j \in E(H)} \frac{1}{\left(d_{i}+d_{j}\right)^{2 \alpha}} \\
& =\left(\frac{2 \Delta^{3 \alpha / 2} \delta^{3 \alpha / 2}}{\Delta^{3 \alpha}+\delta^{3 \alpha}}\right)^{2} M_{2}^{2 \alpha}(H) \chi_{-2 \alpha}(H), \\
\operatorname{ISI}_{\alpha}(H) & \geq \frac{2 \Delta^{3 \alpha / 2} \delta^{3 \alpha / 2}}{\Delta^{3 \alpha}+\delta^{3 \alpha}} \sqrt{M_{2}^{2 \alpha}(H) \chi_{-2 \alpha}(H)} .
\end{aligned}
$$

Lemma 3 gives that the equality in this bound is attained if and only if $2^{\alpha} \delta^{3 \alpha}=2^{\alpha} \Delta^{3 \alpha}$, i.e., $H$ is regular.

The previous theorem is useful, since it establishes functional properties of the variable topological indices $I S I_{\alpha}, M_{2}^{2 \alpha}$ and $\chi_{-2 \alpha}$, and two important invariants of a graph (the maximum degree $\Delta$ and the minimum degree $\delta$ ).

\section{Comparative Studies and Conclusions}

The main goal of the research on topological indices from a theoretical approach is twofold: firstly, to find new relationships among them and, secondly, to obtain optimal bounds (which confirm the validity and relevance of the relations found). Motivated by the studies [12,15], the introduction of a new general index $A_{\alpha}$ was deemed convenient by the authors. This index opens a way of grouping the variable topological indices and analyzing their properties and relations.

In the aforementioned works, only one chemical-computational study is carried out and several numerical relationships between the different topological indices are shown. Our work responds to the need raised in [12,15] in two ways: firstly, by conducting a theoretical-analytical study on the general index $A_{\alpha}$ and, secondly, by obtaining new optimal relationships for that index that involve important invariants of a graph such as measure, order, maximum degree, and minimum degree (see Theorems 1,2 and 3).

It is important to highlight that in the works $[13,14]$, the authors study the variable topological index $M_{1}^{\alpha}$ in tree-type graphs and find optimal bounds for that index in some families of trees. We study the general index $B_{\alpha}$ that contains, as a particular case, the variable topological index $M_{1}^{\alpha}$; furthermore, we find new relationships for all graphs and we obtain optimal bounds associated to other infinite families of graphs (regular and bi-regular graphs).

In [26] the authors study the variable topological index $\chi_{\alpha}$ and generalize the results of [27]. Likewise, they find optimal bounds for $\chi_{\alpha}$ in terms of several invariants of the graph (order, maximum degree and minimum degree). In our research these results are generalized (see Corollaries 1,2 and 3) and new relationships are found between $\chi_{\alpha}$ and other important topological indices (see Theorem 6). It should be noted that all these relationships are optimal, as evidenced by the results.

In [29] the authors generalize the well-known geometric-arithmetic index, for $\alpha \geq 0$ and obtain bounds in terms of invariants of the graph such as: measure, maximum degree and minimum degree. In [30] a new generalization of the geometric-arithmetic index for every real $\alpha$ is shown, and a statistical-computational study is conducted on that index. In this research, the study carried out in [30] is complemented with a theoretical-analytical study of $G A_{\alpha}$ and in the same direction, new optimal bounds that expand the results shown in [29] are obtained (see Corollaries 1,2 and 3 and Theorem 6).

In $[30,31]$ two studies on the variable inverse sum indeg index $I S I_{\alpha}$ are conducted. In the first, from a statistical-computational viewpoint and in the latter, from a spectral perspective. However, neither one considers a theoretical-analytical approach, and that made us follow this perspective in our work, in which optimal relations and bounds are found for the variable inverse sum indeg index (Corollaries 1,2 and 3 and Theorems 4,5 and 6). 
Topological indices have been successfully applied in several branches of science (see, e.g., [4-6]). In this research, we focus on a new general framework, given by two new general topological indices $A_{\alpha}(H)=\sum_{i j \in E(H)} f\left(d_{i}, d_{j}\right)^{\alpha}$ and $B_{\alpha}(H)=\sum_{i \in V(H)} h\left(d_{i}\right)^{\alpha}$. They allow to obtain, with each result on $A_{\alpha}$ (see Theorems 1, 2 and 3) and $B_{\alpha}$ (see Propositions 1, 2 and 3), inequalities for a large family of topological indices. In this general approach, the use of the above mentioned functions ( $f$ and $h$ ) allows us to find new properties of the most important variable topological indices:

- $\quad$ if $f(x, y)=x y$, then $A_{\alpha}$ is the variable second Zagreb index $M_{2}^{\alpha}$;

- if $f(x, y)=x+y$, then $A_{\alpha}$ is the variable sum-connectivity index $\chi_{\alpha}$;

- if $f(x, y)=2 \sqrt{x y} /(x+y)$, then $A_{\alpha}$ is the variable geometric-arithmetic index $G A_{\alpha}$;

- if $f(x, y)=x y /(x+y)$, then $A_{\alpha}$ is the variable inverse sum indeg index $I S I_{\alpha}$;

- $\quad$ if $h(t)=t$, then $B_{\alpha}$ is the variable first general Zagreb index $M_{1}^{\alpha}$.

We obtain, in particular, new optimal bounds and relations between these five variable topological indices in Corollaries 1,2 and 3.

Furthermore, this general approach opens a new line of research in relation to the analytical study of other topological indices and provides a basis for defining new indices with potential application in mathematical chemistry.

Besides, we obtain several optimal bounds of $G A_{\alpha}$ in terms of $I S I_{-\alpha}$ (see Theorems 4 and 5), and two bounds of $I S I_{\alpha}$ involving $M_{2}^{2 \alpha}$ and $\chi_{-2 \alpha}$ in Theorem 6.

Funding: J.M.S. was supported by a grant from Agencia Estatal de Investigación (PID2019-106433GBI00/AEI/10.13039/501100011033), Spain.

Institutional Review Board Statement: Not applicable.

Informed Consent Statement: Not applicable.

Data Availability Statement: Not applicable.

Acknowledgments: We would like to thank the reviewers by their careful reading of the manuscript and their suggestions which have improved the presentation of this work.

Conflicts of Interest: The author declares no conflict of interest.

\section{References}

1. Gutman, I.; Furtula, B. (Eds.) Recent Results in the Theory of Randić Index; Univ. Kragujevac: Kragujevac, Serbia, 2008.

2. Li, X.; Gutman, I. Mathematical Aspects of Randić Type Molecular Structure Descriptors; Univ. Kragujevac: Kragujevac, Serbia, 2006.

3. Li, X.; Shi, Y. A survey on the Randić index. MATCH Commun. Math. Comput. Chem. 2008, 59, 127-156.

4. Gutman, I.; Furtula, B.; Katanić, V. Randić index and information. AKCE Int. J. Graphs Comb. 2018, 15, 307-312. [CrossRef]

5. Estrada, E. Quantifying network heterogeneity. Phys. Rev. E 2010, 82, 066102. [CrossRef] [PubMed]

6. Pineda, J.; Martínez, C.; Mendez, A.; Muños, J.; Sigarreta, J.M. Application of Bipartite Networks to the Study of Water Quality. Sustainability 2020, 12, 5143. [CrossRef]

7. Bollobás, B.; Erdös, P. Graphs of extremal weights. Ars Comb. 1998, 50, 225-233. [CrossRef]

8. Bollobás, B.; Erdös, P.; Sarkar, A. Extremal graphs for weights. Discr. Math. 1999, 200, 5-19. [CrossRef]

9. Randić, M. Novel graph theoretical approach to heteroatoms in QSAR. Chemom. Intel. Lab. Syst. 1991, 10, 213-227. [CrossRef]

10. Randić, M. On computation of optimal parameters for multivariate analysis of structure-property relationship. J. Chem. Inf. Comput. Sci. 1991, 31, 970-980. [CrossRef]

11. Randić, M.; Plavšić, D.; Lerš, N. Variable connectivity index for cycle-containing structures. J. Chem. Inf. Comput. Sci. 2001, 41, 657-662. [CrossRef]

12. Gutman, I.; Tošović, J. Testing the quality of molecular structure descriptors. Vertex-degreebased topological indices. J. Serb. Chem. Soc. 2013, 78, 805-810. [CrossRef]

13. Li, X.; Zheng, J. A unified approach to the extremal trees for different indices. MATCH Commun. Math. Comput. Chem. 2005, 54, 195-208.

14. Li, X.; Zhao, H. Trees with the first smallest and largest generalized topological indices. MATCH Commun. Math. Comput. Chem. 2014, 50, 57-62.

15. Miličević, A.; Nikolić, S. On variable Zagreb indices. Croat. Chem. Acta 2004, 77, 97-101.

16. Nikolić, S.; Miličević, A.; Trinajstić, N.; Jurić, A. On Use of the Variable Zagreb ${ }^{v} M_{2}$ Index in QSPR: Boiling Points of Benzenoid Hydrocarbons. Molecules 2004, 9, 1208-1221. [CrossRef] [PubMed]

17. Drmota, M. Random Trees: An Interplay Between Combinatorics and Probability; Springer: New York, NY, USA, 2009. 
18. Gutman, I. Degree-based topological indices. Croat. Chem. Acta 2013, 86, 351-361. [CrossRef]

19. Gutman, I.; Das, K.C. The first Zagreb index 30 years after. MATCH Commun. Math. Comput. Chem. 2014, 50, 83-92.

20. Gutman, I.; Réti, T. Zagreb group indices and beyond. Int. J. Chem. Model. 2014, 6, 191-200.

21. Singh, M.; Das, K.C.; Gupta, S.; Madan, A.K. Refined variable Zagreb indices: Highly discriminating topological descriptors for QSAR/QSPR. Int. J. Chem. Modeling 2014, 6, 403-428.

22. Rodríguez, J.M.; Sánchez, J.L.; Sigarreta, J.M. On the first general Zagreb index. J. Math. Chem. 2018, 56, 1849-1864. [CrossRef]

23. Zhou, B.N.; Trinajstić, N. On general sum-connectivity index. J. Math. Chem. 2010, 47, 210-218. [CrossRef]

24. Fajtlowicz, S. On conjectures of Graffiti-II. Congr. Numer. 1987, 60, 187-197.

25. Deng, H.; Balachandran, S.; Ayyaswamy, S.K.; Venkatakrishnan, Y.B. On the harmonic index and the chromatic number of a graph. Discrete Appl. Math. 2013, 161, 2740-2744. [CrossRef]

26. Rodríguez, J.M.; Sigarreta, J.M. New Results on the Harmonic Index and Its Generalizations. MATCH Commun. Math. Comput. Chem. 2017, 78, 387-404.

27. Zhong, L. The harmonic index for graphs. Appl. Math. Lett. 2012, 25, 561-566. [CrossRef]

28. Zhong, L.; Xu, K. Inequalities between vertex-degree-based topological Indices. MATCH Commun. Math. Comput. Chem. 2014, 71, 627-642.

29. Eliasi, M.; Iranmanesh, A. On ordinary generalized geometric-arithmetic index. Appl. Math. Lett. 2011, 24, 582-587. [CrossRef]

30. Aguilar-Sánchez, R.; Herrera-González, I.F.; Méndez-Bermúdez, J.A.; Sigarreta, J.M. Computational Properties of General Indices on Random Networks. Symmetry 2020, 12, 1341. [CrossRef]

31. Hafeez, S.; Farooq, R. On generalized inverse sum indeg index and energy of graphs. AIMS Math. 2020, 5, 2388-2411. [CrossRef]

32. Hardy, G.H.; Littlewood, J.E.; Pólya, G. Inequalities, 2nd ed.; Cambridge University Press: Cambridge, UK, 1988.

33. Martínez-Pérez, A.; Rodríguez, J.M.; Sigarreta, J.M. A new approximation to the geometric-arithmetic index. J. Math. Chem. 2018, 56, 1865-1883. [CrossRef] 